\title{
Childhood Injury in Wake County, NC: Local Use of Public Health Surveillance Data
}

\author{
Anna E. Waller*, Steven Lippmann, Amy Ising and Carolyn Crump \\ UNC-Chapel Hill, Chapel Hill, NC, USA
}

\section{Objective}

To utilize secondary data sources to describe childhood injury and prioritize prevention efforts in Wake County, NC.

\section{Introduction}

A local foundation commissioned a project to determine the leading causes of childhood injury in Wake County, NC. Multiple sources of secondary data, including syndromic surveillance data, were used to describe leading causes of childhood injury in the county. ${ }^{1}$

\section{Methods}

Mortality (deaths) were identified in data available online through the NC State Center for Health Statistics (SCHS) and the NC Violent Death Reporting System. Hospital Discharges were identified through the NC SCHS hospital discharge data file and accessed by staff in the Injury and Violence Prevention Branch at NC Division of Public Health (NC DPH). Emergency Department Visits were identified through NC DETECT and accessed by project staff under a Data Use Agreement with NC DPH. Descriptive statistics and crosstabulations were computed using SAS version 9.2 and Microsoft Excel. Injury intent and mechanism were categorized based on ICD10 (mortality) or ICD-9-CM (ED and hospital) external cause codes. Rates were computed using estimates for 2010-2012 as the population denominators.

\section{Results}

To develop a list of the leading causes of injuries among Wake County children ages $0-17$, we rank-ordered the top five injury mechanisms in each of the three data sources (Table 1) and determined the order of the top ten causes based on their occurring in all three, two, or only one of our major data sources. Motor Vehicle Crashes $(M V C)$ (occupant, pedestrian, all) were one of the five leading causes for all three data sources, thus their placement as the $1^{\text {st }}$ and $3^{\text {rd }}$ leading injury causes. MVC-Pedestrian was kept separate as the $3^{\text {rd }}$ leading cause given differences in prevention approaches for occupants versus pedestrians. Assault and Self-Inflicted/Self Harm were in the top five injury causes for both mortality and hospital discharges, thus their placement as $2^{\text {nd }}$ and $4^{\text {th }}$ leading causes. Falls was in the top injury cause for both hospital discharges and ED visits, and the number of fall events was significantly higher than the number of deaths due to Unintentional Suffocation, thus the placement of Falls and Suffocation as the $5^{\text {th }}$ and $6^{\text {th }}$ leading causes, respectively. Burns, Struck By/Against, Natural/Environmental Factors, and Bicycle Injury/Crashes were placed in the $7^{\text {th }}$ through $10^{\text {th }}$ positions because they were among the five leading injury causes for hospital discharges or ED visits only.

More than half the injury related hospital discharges $(58.1 \%)$ and ED visits (58.5\%) were by male children; this was true for all age groups. Children ages 1-4 years had the most injury ED visits, followed by those 10-14 years. Children ages 15-17 years had more injury hospitalizations than any other age group, accounting for $27.7 \%$ of all injury related hospital discharges. The number of injury-related ED visits increased steadily between 2006 and 2012. Nine out of ten injury-related ED visits by Wake County children resulted in being discharged to home $(91.2 \%)$, while $4.5 \%$ resulted in being admitted to the hospital or transferred to another hospital. There were 29 deaths in hospital for injured patients (1.0\%).

\section{Conclusions}

A wealth of information about childhood injury in Wake County, $\mathrm{NC}$, was available through secondary data sources, including syndromic surveillance data. Timely and comprehensive ED visit data collected in NC DETECT proved invaluable to assessing local childhood injury causes. The ranking of childhood injury causes varied greatly between data sources, highlighting the importance of including a variety of data in a community health assessment. Including ICD-9-CM codes in a syndromic surveillance system enables inclusion of these data in health assessments and other retrospective analyses.

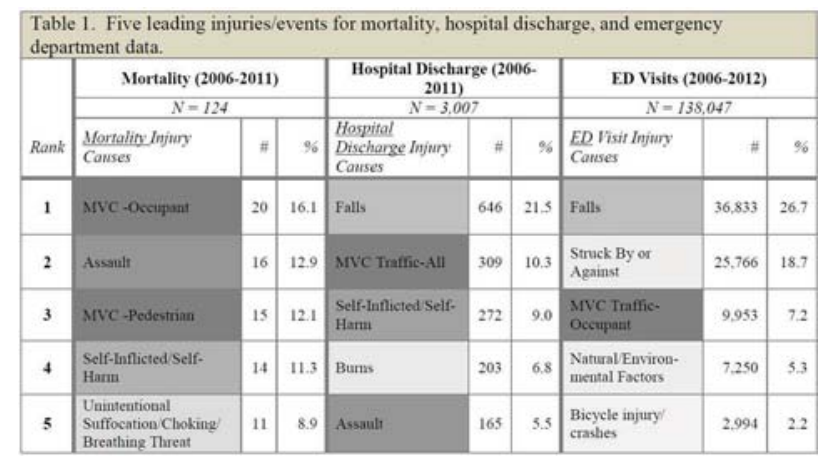

\section{Keywords}

Injury surveillance; Childhood Injury; Community Assessment; Local public health; Secondary data

\section{Acknowledgments}

The ED visit data were obtained from NC DETECT under a Data Use Agreement with the NC Division of Public Health. The NC DETECT Data Oversight Committee takes no responsibility for the scientific validity or accuracy of methodology, results, statistical analyses, or conclusions presented.

\section{References}

1. Crump C, Page R, Letourneau R, Waller A, Lippman S, Ising A (Healthy Solutions, University of North Carolina, Chapel Hill, NC). A Profile of Wake County Childhood Injury \& Injury Prevention. Raleigh (NC): John Rex Endowment; 2014, 102 p.

\section{*Anna E. Waller}

E-mail: awaller@med.unc.edu 